\title{
Estudo comparativo entre reação de Mitsuda e antígenos leucocitários humanos em pacientes hansenianos
}

\author{
Comparative study between the Mitsuda test and the human \\ leukocyte antigens in leprosy patients
}

\author{
Fabiana Covolo de Souza ${ }^{1}$, Elaine Valim Camarinha Marcos ${ }^{1}$, Somei Ura ${ }^{2}$, \\ Paula Araújo Opromolla ${ }^{3}$ e Maria Esther Salles Nogueira ${ }^{1}$
}

\begin{abstract}
RESUMO
Neste estudo, propomos comparar o teste cutâneo de Mitsuda e os alelos HLA-DR2/HLA-DR3 e HLA-DQ1 relacionados com as formas clínicas da hanseníase em 176 pacientes (50 TT, 50 LL e 76 B). Os resultados obtidos não revelaram associação entre reação de Mitsuda e os alelos HLA nas formas clínicas isoladas; no entanto, quando analisados de acordo com a resposta ao teste de Mitsuda, associação significativa foi encontrada entre os pacientes Mitsuda negativos e HLA-DQ1 ( $p=0,002)$. Não foi observada associação entre reação de Mitsuda positiva e alelos HLA-DR2/DR3. Concluímos que existe importante participação do alelo HLA-DQ1 na ausência de resposta ao teste de Mitsuda. Sugerimos estudos mais especificos para este alelo.
\end{abstract}

Palavras-chaves: Hanseníase. HLA. Antígeno de Mitsuda. Mycobacterium leprae.

\section{ABSTRACT}

In this study, we aimed to compare the Mitsuda skin test with the alleles HLA-DR2/HLA-DR3 and HLA-DQ1, in relation to the clinical forms of leprosy in 176 patients (50 TT, $50 \mathrm{LL}$ and $76 \mathrm{~B}$ ). The results obtained did not reveal any association between the Mitsuda reaction and the HLA alleles in the clinical forms isolated. However, when analyzed according to Mitsuda test response, a significant association was found between patients with negative Mitsuda reaction and HLA-DQ1 ( $p=0.002)$. No association was observed between positive Mitsuda reaction and the HLA-DR2/DR3 alleles. We concluded that the allele HLA-DQ1 has an important participation when there is no response to the Mitsuda test. We suggest that more specific studies should be developed on this allele.

Key-words: Leprosy. HLA. Mitsuda antigen. Mycobacterium leprae.

Hanseníase é doença infecciosa crônica, causada pelo Mycobacterium leprae (M. leprae $)^{520}$, com incidência anual de aproximadamente 400.000 casos no mundo ${ }^{27}$ e 30.000 no Brasil ${ }^{18}$. É considerada grave problema de saúde pública e social, devido às seqüelas físicas e deformidades permanentes que o paciente manifesta ${ }^{23}$.

As diferentes formas clínicas da doença expressam o grau de imunidade específica do hospedeiro contra o bacilo ${ }^{14}$. Fundamentado nos critérios estabelecidos por Ridley \& Jopling ${ }^{21}$, é classificada em cinco formas polares e interpolares: a forma tuberculóide (TT) com resposta imune eficiente e a virchoviana (LL) com resposta ineficaz. As formas interpolares representam o grupo dimorfo (BB, BT, BL), que apresentam manifestações clínicas e imunológicas variáveis entre os pólos TT e LL7.
A reação cutânea de Mitsuda consiste em um teste oficialmente aceito como critério de classificação e prognóstico da hanseníase e constitui parâmetro imunológico para avaliar a resposta tardia a uma sequiência de eventos que se seguem à fagocitose dos bacilos contidos na suspensão, pelos histiócitos ${ }^{10}$. Através deste, é possível avaliar a resposta imune celular nos indivíduos, resposta esta, que está intimamente relacionada à progressão e manifestação clínica da doença ${ }^{213}$.

No consenso geral, pacientes TT são definidos como Mitsuda positivos e LL como Mitsuda negativos. Os grupos interpolares apresentam reações variáveis dependendo do grau de imunidade do paciente.

O complexo HLA, na hanseníase, começou a ser estudado na tentativa de elucidar os mecanismos de suscetibilidade ou

1. Equipe Técnica de Imunologia, Instituto Lauro de Souza Lima, Bauru, SP. 2. Divisão de Ensino e Pesquisa, Instituto Lauro de Souza Lima, Bauru, SP. 3. Núcleo de Apoio e Pesquisa, Instituto Lauro de Souza Lima, Bauru, SP.

Endereço para correspondência: Ms Fabiana Covolo de Souza. Instituto Lauro de Souza Lima. Rodovia Comandante João Ribeiro de Barros, km 225/226, Caixa Postal 3021, 17034-971 Bauru, SP.

Tel: 55 14 3103-5913, Fax: $55143103-5914$

e-mail: fsouza@ilsl.br

Recebido para publicação em 13/3/2006

Aceito em 2/4/2007 
de resistência para a doença, uma vez que estes alelos atuam de forma direta na resposta imune, através da apresentação do peptídeo antigênico ao linfócito $\mathrm{T}^{68}$. Associações positivas entre TT e alelos HLA-DR2, HLA-DR3 e entre LL e o alelo HLA-DQ1 têm sido descritas em diferentes populações do mundo ${ }^{49} 17242526$.

Estudos ${ }^{15}{ }^{19}$ têm demonstrado que o complexo HLA, na hanseníase, participa na modulação e manifestação das formas clínicas da doença, e não influencia na suscetibilidade para a infecção propriamente dita. Assim, tanto a reação de Mitsuda como o complexo HLA atuam no prognóstico da hanseníase, ou seja, indivíduos Mitsuda positivos portadores do alelo HLA-DR2 ou HLA-DR3 teriam maior probabilidade de desenvolver a forma TT; $\mathrm{e}$ os Mitsuda negativos portadores do alelo HLA-DQ1, a forma LL.

Fundamentado na premissa supracitada e na falta de dados na literatura, este estudo foi proposto, com o objetivo de verificar a concordância entre os resultados da reação de Mitsuda comparados aos alelos HLA de classe II (lócus DRB1* e DQB1*) nas diferentes formas clínicas da hanseníase visando à melhor compreensão dos mecanismos imunológicos envolvidos na patogenia desta doença.

\section{MATERIAL E MÉTODOS}

Pacientes. Participaram do estudo 176 pacientes, classificados de acordo com os critérios estabelecidos por Ridley \& Jopling ${ }^{21}$, distribuídos em TT ( $=50)$, BT $(n=22)$, BB $(n=29)$, BL $(n=25)$ e $\mathrm{LL}(\mathrm{n}=50)$ diagnosticados no Serviço de Dermatologia do Instituto Lauro de Souza Lima (ILSL) de Bauru-SP.

Aspectos éticos. Todos os pacientes foram informados e concordaram em participar da pesquisa assinando o Termo de Consentimento Informado Livre e Esclarecido, de acordo com a Resolução 196 do Conselho Nacional de Saúde. O projeto foi aprovado pela Comissão Científica e pelo Comitê de Ética em Pesquisa do ILSL, sob n ${ }^{0} 0202$.

Teste de Mitsuda ${ }^{12}$. Suspensão injetada na face anterior do braço, por via intradérmica, $0,1 \mathrm{ml}$ na concentração de $6,0 \times 10^{7}$ M. leprae/ml, preparada na própria instituição. A leitura clínica da reação foi realizada 28 dias após a injeção do antígeno. Calculou-se a média aritmética dos dois maiores diâmetros do nódulo, conforme critérios propostos pelo Congresso Mundial de Leprologia ${ }^{10}$, descritos a seguir: Negativo (-) ausência de resposta; Duvidoso ( \pm ) infiltração com diâmetro < 3mm; Positivo (+) infiltração com diâmetro entre 3 e $5 \mathrm{~mm}$; Positivo $(++)$ infiltração com diâmetro > 5mm; Positivo $(+++)$ infiltração com diâmetro $\geq 10 \mathrm{~mm}$ e ulcerado.

Tipificação dos antígenos leucocitários humanos. HLA de classe II lócus DRB1* (DRB1*01-16) e DQB1* (DQB1*0109) foram realizadas através da extração de DNA pela técnica de Salting out $^{11}$ e pelo método de PCR-SSP, utilizando-se kits comerciais de baixa resolução da marca One-Lambda-USA.

Análise estatística. Teste do Qui-quadrado foi utilizado para detecção de associação entre os resultados do teste de Mitsuda e alelos HLA. As análises estatísticas foram realizadas ao nível de 5\% de significância com o emprego do pacote estatístico SPSS 11.0 para Windows.

\section{RESULTADOS}

0 grupo TT revelou $100 \%$ de positividade para o teste de Mitsuda, o padrão de leitura foi positivo ${ }^{+}(16 \%)$; positivo ${ }^{++}$ (68\%) e positivo ${ }^{+++}(16 \%)$. 0 diâmetro da induração variou entre $4,0 \mathrm{~mm}$ a $18,0 \mathrm{~mm}$ e o valor médio dos resultados $7,5 \mathrm{~mm}( \pm 2,6)$. Os alelos HLA-DR2 e HLA-DR3 neste grupo representaram 46\% ( $\mathrm{p} \geq 0,005)$ da amostra (Tabela 1).

Pacientes LL demonstraram 100\% de negatividade ao teste de Mitsuda. 0 alelo HLA-DQ1 representou 74\% ( $\mathrm{p} \geq 0,005)$ nesta forma (Tabela 2).

\begin{tabular}{|c|c|c|c|}
\hline \multirow{3}{*}{ Fenótipo } & \multirow{3}{*}{ Alelo HLA } & \multirow{2}{*}{\multicolumn{2}{|c|}{$\begin{array}{c}\text { Presença do alelo } \\
\text { pacientes com o alelo }\end{array}$}} \\
\hline & & & \\
\hline & & $\mathrm{n}^{\mathrm{0}}$ & $\%$ \\
\hline \multirow[t]{2}{*}{ DR2 } & DRB1*15 & 12 & 24,0 \\
\hline & $\mathrm{DRB} 1 * 16$ & 2 & 4,0 \\
\hline \multirow[t]{2}{*}{ DR3 } & DRB1*17 & 4 & 8,0 \\
\hline & DRB1*18 & 4 & 8,0 \\
\hline $\mathrm{DR} 2+\mathrm{DR} 3$ & DRB1*15 + DRB $1 * 17$ & 1 & 2,0 \\
\hline Outros marcadores & & 27 & 54,0 \\
\hline Total & & 50 & 100,0 \\
\hline
\end{tabular}

Tabela 2- Distribuição da presença de alelos HLA-DQ1(HLA-DQB1*05 e HLA-DQB1*06) nos 50 pacientes $L L$ com reação de Mitsuda negativa.

\begin{tabular}{llll}
\hline \multirow{2}{*}{ Fenótipo } & Alelo HLA & \multicolumn{2}{c}{ Presença do alelo } \\
\cline { 3 - 4 } & & \multicolumn{2}{c}{ pacientes com 0 alelo } \\
\cline { 3 - 4 } $\mathrm{n}^{0}$ & $\%$ \\
\hline DQ1 & DQB1*05 & 17 & 34,0 \\
& DQB1*06 & 14 & 28,0 \\
& DQB1*05 e DQB1*06 & 6 & 12,0 \\
\hline Outros marcadores & & 13 & 26,0 \\
\hline Total & & 50 & 100,0 \\
\hline
\end{tabular}

No grupo B, o padrão da leitura clínica do teste foi negativo $(48,7 \%)$; duvidoso $(5,2 \%)$; positivo $^{+}(30,3 \%)$ e positivo ${ }^{++}$ $(15,8 \%)$. 0 diâmetro da induração variou entre negativo a $7,5 \mathrm{~mm}$, representando uma média de $2,4 \mathrm{~mm}$. $( \pm 2,5)$. Neste grupo, não foi possível realizar a comparação entre reação de Mitsuda e alelos HLA uma vez que não foi encontrado nenhum alelo HLA associado a esta forma clínica da doença.

Quando a análise dos resultados foi realizada, segundo a resposta ao teste de Mitsuda e independente da forma clínica, dos 176 pacientes estudados, verificou-se positividade para 0 teste de Mitsuda em 85 (48,3\%), destes, 43,5\% possuíam o alelo HLA-DR2 ou HLA-DR3 não confirmando associação entre reação de Mitsuda positivo e alelo HLA-DR2/DR3 pelo teste do Qui-quadrado ( $\mathrm{p} \geq 0,05)$. Contudo, 91 pacientes $(51,7 \%)$ que demonstraram negatividade ao teste de Mitsuda, 65,9\% possuíam 0 alelo HLA-DQ1. Verificou-se associação entre reação de Mitsuda negativo e alelo HLA-DQ1 em nível descritivo de 0,002 , pelo teste do Qui-quadrado (Tabela 3). 
Tabela 3- Distribuição de 85 pacientes hansenianos Mitsuda positivos quanto à presença de alelo HLA-DRB1*15 ou HLA-DRB1*16 (HLA-DR2), HLA-DRB1*17 ou HLA-DRB1*18 (HLA-DR3) e de 91 pacientes Mitsuda negativos quanto à presença do alelo HLA-DQB1*05 ou HLA-DQB1*06 (HLA-DQ1).

\begin{tabular}{|c|c|c|c|c|c|c|}
\hline \multirow[b]{3}{*}{ Reação de Mitsuda } & \multicolumn{5}{|c|}{ Presença de alelo HLA nos pacientes } & \multirow[b]{3}{*}{ Total } \\
\hline & \multicolumn{2}{|c|}{$\operatorname{sim}$} & \multicolumn{2}{|c|}{ não } & \multirow[b]{2}{*}{$\mathrm{p}^{*}$} & \\
\hline & $\overline{\mathrm{n}^{0}}$ & $\%$ & $\mathrm{n}^{0}$ & $\%$ & & \\
\hline Positivo $^{1}$ & 37 & 43,5 & 48 & 56,5 & 0,233 & 85 \\
\hline Negativo $^{2}$ & 60 & 65,9 & 31 & 34,1 & 0,002 & 91 \\
\hline Total & & & & & & 176 \\
\hline
\end{tabular}

* teste de Qui-quadrado, gl = 1 .

1. em relação a HLA-DR2 e HLA-DR3

2. em relação a HLA-DQ1.

\section{DISCUSSÃo}

No presente estudo, avaliou-se a concordância entre a reação de Mitsuda e alelos HLA-DR2/DR3 e HLA-DQ1 em 176 pacientes portadores de hanseníase classificados segundo os critérios estabelecidos por Ridley \& Jopling ${ }^{21}$.

Os resultados da reação de Mitsuda, no grupo de pacientes estudados, concordaram com a descrição da literatura na qual evidencia que pacientes TT são Mitsuda positivos, LL Mitsuda negativos e os B, geralmente, fracamente positivos ou negativos ${ }^{22}$.

Quando comparados os resultados da reação de Mitsuda com os alelos HLA-DR2 e HLA-DR3 na forma TT, assim como HLA-DQ1 na forma LL, não foi possível estabelecer relação significativa entre estes resultados.

Cabe ressaltar que no grupo $\mathrm{B}$, até o momento, não existe descrito na literatura nenhum alelo HLA associado a esta forma da doença, portanto para este grupo, inicialmente foi realizado um estudo de associação entre alelos HLA e B. 0 resultado deste estudo não demonstrou nenhuma associação dos alelos HLA neste grupo, impossibilitando a comparação com a reação de Mitsuda.

Baseado nas descrições de Ottenhoff ${ }^{17}$ e Britton ${ }^{3}$ que postularam o alelo HLA-DR2 e/ou HLA-DR3 relacionado à indução de uma resposta imune celular eficiente contra o $M$. leprae e reação de Mitsuda positiva, o alelo HLA-DQ1relacionado a resposta imune deficiente e reação de Mitsuda negativa, e a falta de concordância observada nos resultados descritos inicialmente neste estudo, uma nova análise foi proposta, na qual os pacientes foram separados de acordo com a resposta a teste de Mitsuda.

No grupo de pacientes com a reação de Mitsuda positiva persistiu a falta de relação com os alelos HLA, discordando da literatura que sugere a participação dos alelos HLA-DR2 e HLADR3 na resposta positiva da reação de Mitsuda ${ }^{317}$.

Ottenhoff e cols ${ }^{16}$ em estudo envolvendo pacientes BT reacionais e não reacionais, verificaram associação do HLA-DR3 com a resposta fortemente positiva da reação de Mitsuda no grupo reacional. Segundo os autores, a frequiência elevada do HLA-DR3 sugere a participação deste na imunidade celular dos pacientes que sofrem o surto reacional, fato também observado por Marcos e cols ${ }^{8}$, em estudo com pacientes TT e BB reacionais.

A discordância entre os resultados obtidos em nosso estudo com os dados da literatura pode ser justificada nas características peculiares que envolvem o complexo HLA, como o polimorfismo, herança genética, a variação da frequiência dos alelos observada entre as diferentes etnias ${ }^{15}$, além do pequeno número de pacientes envolvidos. Sendo assim, estudos neste contexto devem ser analisados individualmente para que suas particularidades sejam percebidas ${ }^{1}$.

Quando analisados os grupos de acordo com a resposta ao teste de Mitsuda, no grupo com reação de Mitsuda negativa, foi possível detectar associação estatisticamente significante em nível descritivo de 0,002, pelo teste do Qui-quadrado, sugerindo o envolvimento do alelo HLA-DQ1 na resposta imune deficiente contra o M. leprae, corroborando os dados da literatura ${ }^{315}$.

Considerando que antígenos iguais são reconhecidos tanto por indivíduos que adoecem quanto por indivíduos que nunca irão desenvolver a doença e que diferenças são observadas na habilidade desses indivíduos em responder aos mesmos antígenos $^{17}$ infere-se que possa ser o peptídeo antigênico apresentado pelo alelo HLA-DQ1 o responsável pela ausência de imunidade celular observada nos pacientes não respondedores ao teste de Mitsuda.

Fundamentado nos resultados obtidos, foi possível verificar o perfil de resposta imune celular específica para o M. leprae e a distribuição dos alelos HLA sob os aspectos considerados: formas clínicas e positividade ao teste de Mitsuda. Todavia, os estudos devem ser ampliados para que se possa avaliar e compreender, com maior fidelidade, mecanismos como os de imunoregulação, fisiopatologia e evidenciar a participação destes componentes na infecção hansênica, para que diagnósticos mais precisos sejam firmados e novas terapêuticas sejam instituídas.

\section{REFERÊNCIAS}

1. Alves C, Vieira N, Meyer I, Alves CO, Toralles MBP, Oliveira MFSP. Antígenos de histocompatibilidade humanos e dermatologia: da pesquisa para a prática clínica. Anais Brasileiros de Dermatologia 81: 65-73, 2006.

2. Beiguelman B. A reação de Mitsuda oitenta anos depois. Hansenologia Internationalis 24:144-161, 1999 .

3. Britton WJ. Leprosy 1962-1992: immunology of leprosy. Transactions of the Royal Society of Tropical Medicine and Hygiene 87:508-514, 1993.

4. Izumi S, Sugiyama K, Matsumoto Y, Ohkawa S. Analysis of the immunogenetic background of Japanese leprosy patients by the HLA system. Vox sanguinis 42: 243-247, 1982.

5. Jopling WH, McDougall AC, Bakos L. Manual da Hanseníase. $4^{\mathrm{a}}$ edição, Editora Atheneu, Rio de Janeiro, 1991.

6. Lagrange $\mathrm{PH}$, Abel L. Susceptibilité génétique à la lèpre chez l'homme. Acta Leprologica 10: 11-27, 1996.

7. Lastória JC, Opromolla DVA, Fleury RN, Rabermann F, Curi PR. Serial Mitsuda tests for identification of reactional tuberculoid and reactional boderline leprosy forms. International Journal of Leprosy 66: 190-200, 1998.

8. Marcos EVC. Imungenética. In: Noções de Hansenologia. $2^{\text {a }}$ edição, Centro de Estudos “Dr. Reynaldo Quagliato”. Instituto Souza Lima, Bauru, p.43-46, 2000. 
9. Marcos EVC, Souza FC, Ura S, Opromolla DVA. Estudo de associação entre antígenos HLA e reação hansênica tipo 1 ulcerada. Anais Brasileiros de Dermatologia 75: 283-290, 2000.

10. Memória Association International de la Lepra. In: Congresso Internacional de Leprologia 6, Madrid p.1344, 1953.

11. Miller SA, Dykes DD, Polesky HF. A simple salting out procedure for extracting DNA from human nucleated cells. Nucleic Acids Research 16:1215, 1988.

12. Ministério da Saúde. Secretaria de Políticas de Saúde. Guia para Utilização de Medicamentos e Imunobiológicos na área de Hanseníase. Centro de Documentação do Ministério da Saúde, Brasília, 2000.

13. Mitsuda K. On the value of a skin reaction to a suspension of leprous nodules. International Journal of Leprosy 21:347-358, 1953.

14. Opromolla DVA. Manifestações Clínicas e Reações. In: Noções de Hansenologia. $2^{a}$ edição, Centro de Estudos "Dr. Reynaldo Quagliato". Instituto Lauro de Souza Lima, Bauru, p.51-58, 2000.

15. Ottenhoff THM. Immunology of leprosy. New developments. Tropical Geographical Medicine 46: 72-80, 1994.

16. Ottenhoff THM, Converse GB,Vries RRP. HLA Antigens and Neural Reversal Reaction in Ethiopian Boderline Tuberculoid Leprosy Patients. International Journal of Leprosy 55: 261-66, 1987.

17. Ottenhoff THM, Gonzalez NM,Vries RR, ConvitJ, Van Rood JJ. Association of HLA specificity LB-E12 (MB1, DC1, MT1) with lepromatous leprosy in a Venezuelan population. Tissue antigens 24: 25-29, 1984.
18. Pan American Health Organization. Status of Leprosy in the Region of the Americas. Disponível em: http://www.paho.org. Acesso em 16 de fevereiro de 2007.

19. Petzel-Erler ML. Genetics of the immune response and disease susceptibility. Ciência e Cultura 51: 199-211, 1999.

20. Rees RJW. The microbiology of leprosy. In: Hastings RC (ed) Leprosy. $4^{\text {th }}$ edition Churchil. Livingstone, Edinburg, p.49-83, 1994.

21. Ridley DS, Jopling WH. Classification of Leprosy According to Immunity. A fivegroup system. International Journal of Leprosy 34: 255-273, 1966.

22. Rotberg A. Some aspects of immunity on leprosy and their importance in epidemiology, pathogenesis and classification of forms of the disease. Based on 1529 lepromin-tested cases. Revista Brasileira de Leprologia 5:45-97, 1937.

23. Talhari S, Neves RG. Hansenologia. Calderaro Ltda, Manaus, 1984.

24. Van Eden W, Mehra NK, Vaidya MC, D’Amarco J, Schreuder GMTh, Van Rood JJ. HLA and sporadic tuberculoid leprosy: a population study in Maharashta, India. Tissue antigens 18: 189-194, 1981.

25. Van Eden W, Vries RR, D’Amaro J, Schreuder I, Leiker DL, Van Rood JJ. HLA-DR associated genetic control of the type of leprosy in a population from Surinam. Human Immunology 4: 343-350, 1982.

26. Visentainer JEL, Tsuneto LT, Serra MF, Peixoto PR, Petzel-Erler ML. Association of leprosy with HLA-DR2 in a Southern Brazilian population. Brazilian Journal of Medical and Biological Research 30: 51-59, 1997.

27. World Health Organization. Elimination of Leprosy as a Public Health Problem. Disponível em: http://www.who.int/lep/. Acesso em 16 de fevereiro de 2007. 